\title{
Prebióticos y su efecto en la biod sponibilidad del calcio
}

\author{
Prebiotics and their effect on \\ calcium bioavailability
}

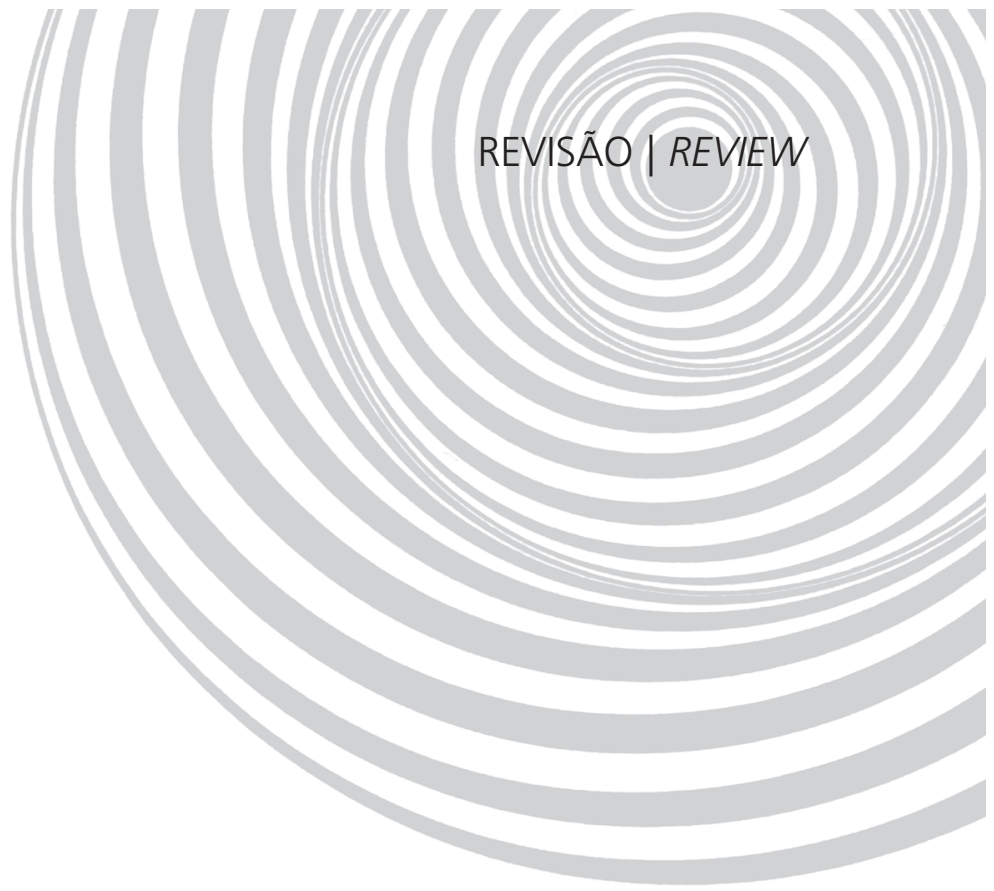

Ivana LAVANDA'

Susana Marta Isay SAAD2

Alexandre Rodrigues $\mathrm{LOBO}^{1}$

Célia COLLI ${ }^{1}$

\section{RE S U M E N}

En la búsqueda de medidas de prevención de enfermedades crónicas no trasmisibles, se piensa en los prebióticos como una forma efectiva, saludable y relativamente barata para la optimización de la absorción de Ca. El aumento de la expectativa de vida de la población y el incremento de enfermedades como la osteoporosis traen graves consecuencias a la salud del individuo, acarreando importantes desembolsos económicos. En esta revisión se condensa el conocimiento presente sobre el efecto fisiológico del consumo de carbohidratos prebióticos y su posible interferencia en la biodisponibilidad del Ca, como también las posibilidades de su empleo en estrategias para combatir las deficiencias del mineral. Se elaboró una recopilación de los estudios originales realizados con mayor impacto en el tema, dando preferencia a los publicados en los últimos 6 años. Utilizamos las bases de datos PubMed, Lilacs e SciELO, usando las palabras claves calcio, prebióticos e probióticos.

Palabras clave: Calcio. Dietética. Minerales. Polisacáridos. Prebióticos.

A B S T R A C T

The increased life expectancy and prevalence of non-communicable chronic diseases have serious health and economic consequences on the population. Among the useful tools for preventing osteoporosis, prebiotics are thought to be an effective, healthy and relatively cheap way of optimizing calcium absorption. The objective of this review was to summarize the present knowledge on the physiological effects of prebiotic carbohydrate consumption and its possible interference with calcium bioavailability. The possibility of using prebiotics to fight calcium deficiency was also assessed. The keywords calcium, prebiotics and probiotics were used to search the PubMed, Lilacs and SciELO databases for the most significant original studies on the subject published in the last 6 years.

Indexing terms: Calcium. Dietetics. Minerals. Polysaccharides. Prebiotics.

${ }^{1}$ Universidade de São Paulo. Departamento de Tecnología Bioquímico-Farmacéutica. São Paulo, SP, Brasil.

${ }^{2}$ Universidade de São Paulo, Faculdade de Ciências Farmacêuticas, Departamento de Alimentos e Nutricão Experimental. Av. Lineu Prestes, 580, Bloco 14, 05508-000, São Paulo, SP, Brasil. Correspondência para/Correspondence to: C. COLLI. E-mail: <cecolli@usp.br>. 


\section{INTRODUCCIÓ N}

En la actualidad se han evidenciado diversos efectos interesantes atribuidos a los llamados "alimentos funcionales", especialmente a los prebióticos y probióticos. Estos alimentos procuran optimizar las funciones fisiológicas del individuo, reduciendo el surgimiento de enfermedades.

Recordando las últimas definiciones, se entiende como probiótico a los microorganismos vivos que, administrados en cantidades adecuadas, confieren beneficios a la salud del húesped ${ }^{1,2}$. Por otro lado, se define como prebióticos a los ingredientes selectivamente fermentables que permiten modificaciones específicas en la composición y/o en la actividad de la microflora gastrointestinal y que otorgan beneficios a la salud del huesped ${ }^{3}$. El ler encuentro de la Asociación Científica Internacional de Probióticos y Prebióticos (ISAPP) realizada en 2002 redefinió el concepto de prebiótico como las sustancias no digeribles que proveen un beneficio fisiológico al huésped por estimulación selectiva del crecimiento o actividad de un limitado número de bacterias endógenas. Esta definición amplía el uso de la definición original, que relaciona los prebióticos sólo con las bacterias del colon. Por lo tanto, el poder de alcance del prebiótico ahora considera la posibilidad de que actúe en otras áreas del tracto gastrointestinal como la zona de la boca, el estómago y el intestino delgado, y otros sitios como la vagina y la piel ${ }^{4}$.

Vale esclarecer también que un producto simbiótico es aquel en el que se combinan un probiótico y un prebiótico. La interacción entre probiótico y prebiótico in vivo puede favorecerse por la adaptación del probiótico al sustrato prebiótico, anterior al consumo 5,6 .

Los alimentos funcionales forman parte de un concepto de alimentos lanzado por Japón en la década del 1980 a través de un programa gubernamental (FOSHU), que tenía como objetivo desarrollar alimentos saludables para una población que envejecía y cuya expectativa de vida era cada vez mayor ${ }^{7}$. Son alimentos que proveen la oportunidad de combinar productos comestibles con moléculas biológicamente activas, como estrategia para corregir disturbios metabólicos ${ }^{8}$.

A través de la actividad de la microflora intestinal y su modulación con la dieta, el colon juega el papel principal en la nutrición del huésped, siendo el primer blanco de la intervención dietética9 ${ }^{9}$.

\section{Efectos de los prebióticos}

Diversos sustratos no digeribles, ya identificados como prebióticos ${ }^{9,10}$, promueven el crecimiento de los lactobacilos y las bifidobacterias.

Para que el sustrato dietético pueda ser clasificado como prebiótico se requieren por lo menos tres características:

No debe ser hidrolisado ni absorbido en el estómago o en el intestino delgado; su estímulo debe ser selectivo para las bacterias benéficas (bifidogénicas y lactobacilos), y su fermentación debe inducir a un efecto luminal y sistémico dentro del huésped.

En la actualidad existen sólo 2 ingredientes alimentarios que cumplen con todos los criterios necesarios para ser considerados prebióticos: la inulina y los trans-galactooligosacáridos (TOS). La inulina tiene moléculas de fructosa con uniones $\beta 2-1$, las cuales son indigeribles para el intestino humano. La inulina de la achicoria está compuesta por una mezcla de oligosacáridos y polímeros, en los cuales el grado de polimerización varía entre 6 a 20 unidades de glucosa. Los transgalactooligosacáridos son una mezcla de oligosacáridos derivados de la lactosa por transglucosidación enzimática. Las mezclas de los productos dependen de las enzimas utilizadas y las condiciones de la reacción. Generalmente se trata de oligosacáridos a partir de tri- o pentascaridos, con uniones $\beta$ 1-6, $\beta$ 1-3 y $\beta$ 1-411.

Parte de la lactosa que no es digerida llega al intestino y se comporta como fibra dietética, aumentando el contenido de agua de las heces y 
reduciendo el tiempo de tránsito intestinal en sujetos constipados. Cada vez más efectos prebióticos de la lactosa recibieron atención. Además de los derivados de la lactosa no digerida, la lactulosa, el lactitol, el ácido lactobiónico y los galacto-oligosacáridos (GOS) y la tagatosa, tienen los mismos efectos que la lactosa que escapa de la digestión ${ }^{12}$.

La Inulina, Los Fructooligosacáridos (FOS), los trans-GOS, la lactulosa y el almidón resistente, cuando consumidos en relativas pequeñas cantidades (5-20g/día) muestran, en estudios realizados en humanos, un estímulo para el crecimiento tanto de bifidobaterias como lactobacilos ${ }^{10,13}$. Muchas frutas y vegetales contienen oligosacáridos prebióticos como los FOS, entre ellos la cebolla, el ajo, la banana, los espárragos, la alcachofa, la achicória y el yacón. Pedreschi et al..$^{14}$ demostró in vitro que los FOS provenientes del yacón son potencialmente fermentados; el yacón (Polymnia sonchifolia) fue clasificado como un nuevo tipo de prebiótico. El mismo efecto bifidogénico fue comprobado en la inulina extraída de la achicoria (Cichorium intybus $L$. $)^{15}$. No obstante, lo más probable es que el contenido de FOS en los alimentos es demasiado bajo para ejercer un efecto significativo. Serían necesarios valores cercanos a 8gr/día para elevar la multiplicación de bifidobacterias en el intestino ${ }^{9}$.

El almidón resistente ha recibido mucha atención tanto por su potencial benéfico a la salud (similar a la fibra soluble) como por sus propiedades funcionales (aumenta el crecimiento de la microflora, tiene bajo índice glucémico y disminuye los niveles de colesterol). Los estudios experimentales realizados en cerdos y en humanos revelaron un cambio en el tiempo del tránsito intestinal y la producción de grandes cantidades de AGCC, lo que sugiere la posible interacción del almidón resistente con las bacterias intestinales ${ }^{16}$.

Los Oligosacáridos (OS) de la leche humana son un componente de relevancia, siendo el tercer componente sólido después de la lactosa y la grasa. Estos OS no se digieren en el intestino delgado y llegan intactos al colon, donde son fermentados por la microflora, la cual estimula su crecimiento. Indirectamente esto provoca una modificación del $\mathrm{pH}$, así como la liberación de distintas sustancias y la estimulación de la respuesta inmune luminal ${ }^{17}$.

El enlace glicosídico entre los monosacáridos es esencial para determinar la selectividad de la digestión y la fermentación en el intestino grueso. A pesar de esto, aún no se ha podido comprender adecuadamente el mecanismo por el cual los oligosacáridos que tienen función prebiótica se metabolizan selectivamente por los miembros benéficos de la microflora. Hay dos paradigmas generales sobre el metabolismo de los prebióticos:

$1^{\circ}$ ) El más documentado es que los microorganismos probióticos, especialmente las bifidobacterias, contienen exo-glicosidasas. Tales enzimas actúan hidrolizando los monosacáridos del extremo no reductor de los oligosacáridos, y entonces son tomados por la célula. Este mecanismo se ha demostrado en la Bifidobacterium infantis, que posee actividad beta fructofuranosidasa ${ }^{18}$, y en el Lactobacilus paracasei $1195^{19}$. En la actualidad, la mayoría de los prebióticos tienen un Grado de Polimerización (GP) pequeño, a excepción de la inulina. Ya que los oligosacáridos deben ser hidrolizados antes de ser incorporados a la célula para sufrir el proceso fermentativo, se supone que a mayor GP más distal es la duración de su efecto prebiótico (ej. la inulina). Es interesante observar que en las bacterias que crecen con inulina se detectó la actividad de una inulinasa extracelular, pero las bacterias que sólo crecen con FOS es probable que no tengan esta enzima $a^{9,20}$.

$\left.2^{\circ}\right)$ El otro mecanismo alternativo es el uptake (el pasaje para dentro de la célula) de oligosacáridos intactos por algunos probióticos, seguido por la hidrólisis intracelular. Hay evidencias de este mecanismo en algunas especies ${ }^{21}$. Las bifidobacterias también producen inulinasas 
intracelulares, necesarias para hidrolizar las uniones beta (2-1) entre las fructosas ${ }^{22}$. En un estudio realizado por Kaplan y Hutkins ${ }^{19}$ se observó que tanto el uptake de los FOS como su hidrólisis fueron inducidos por la sacarosa $u$ oligosacáridos mayores, y a su vez, fueron inhibidos por productos de su hidrólisis (glucosa o fructosa), indicando que los azúcares pueden ser transportados por el mismo sistema que los FOS.

\section{Absorción de Ca y prebióticos}

La osteoporosis es una enfermedad caracterizada por una insuficiencia de masa ósea y un deterioro estructural del tejido óseo que provoca un aumento de la susceptibilidad a las fracturas. La pérdida ósea es principalmente consecuencia del envejecimiento normal, pero también puede ocurrir debido a un deterioro en el desarrollo de la masa ósea máxima o por la pérdida excesiva en la adultez ${ }^{23}$. Es sabido que la osteoporosis está aumentando en el mundo y se predice un incremento del 300\% para el 2050 en Asia. La prevención de este problema de salud pública traería considerables beneficios sociales y económicos. La maximización de la masa ósea durante la adolescencia puede posponer, y quizás prevenir, las fracturas en edades más avanzadas debidas a la osteoporosis ${ }^{24,25}$.

El Ca y el Mg son los nutrientes más importantes para lograr el máximo pico de masa ósea durante el crecimiento, reduciendo los riesgos de fracturas en edades posteriores. Normalmente sólo el 30\% del Ca de la dieta es absorbido por el cuerpo y depositado en el hueso. Mejorando esta absorción, probablemente se lograría evitar futuras fracturas óseas ${ }^{21}$. En el caso del Ca y el $\mathrm{Mg}$, el intestino delgado es el lugar de absorción inicialmente establecido, aunque recientes estudios en ratas indicaron que estos minerales pueden absorberse en el intestino grueso (ciego y colon) $(14 ; 25 ; 26 ; 28)$. Se ha comprobado que el consumo de alimentos funcionales que contienen prebióticos (inulina y FOS) puede contribuir al aumento en la biodisponibilidad de estos minerales.

Hay estudios que demuestran que los prebióticos no sólo aumentan la absorción de $\mathrm{Ca}$ sino también su depósito en el hueso de la rata $^{24,27,32-36}$.

El Ca es absorbido por dos mecanismos:

- Activo o ruta transcelular (cerca del $20 \%$ ). El Ca luminal entra a la célula por los canales de Ca TRPV6 (CaT1/EcaC2) que se encuentran en la membrana apical, es secuestrado por una proteína transportadora intracelular, la calbindina (que necesita la presencia de vitamina D), y liberado por extrusión por una ATPasa por la membrana basolateral de los enterocitos. Esta ruta es bastante importante cuando la ingesta de Ca es baja, ya que la expresión de CaT1 depende de los niveles de vitamina $\mathrm{D}$ y de la necesidad de $\mathrm{Ca}$ del organismo.

- Pasivo o ruta paracelular (cerca del 80\%). El Ca es absorbido a través de las tensas junciones o ensambles celulares. Se trata de un mecanismo insaturable que depende de la concentración de Ca intraluminal, ocurre en toda la longitud del intestino delgado y grueso y es independiente de la vitamina D. Esta ruta puede ser susceptible a la manipulación de los sustratos fermentables $25,28-31$.

Un mecanismo para mejorar la eficacia de la absorción de minerales inducida por los prebióticos podía ser la distribución de los grados de polimerización. Los ingredientes de baja polimerización tales como los FOS son más activos en el colon proximal, donde pueden servir como inductores de la fermentación bifidogénica, mientras que las moléculas de cadena más larga podrían tener su efecto en la parte distal (donde pueden mantener la actividad metabólica de la microflora). Combinar ambos tipos de componentes en un producto puede ofrecer un efecto sinérgico sobre la absorción del $\mathrm{Ca}$, ya que dicha absorción ocurre a lo largo de todo el intestino ${ }^{30}$. 


\section{Hipótesis}

El surgimiento de varias hipótesis permite explicar el efecto de los FOS en la absorción y retención de $\mathrm{Ca}$. Entre estas hipótesis se incluyen:

- Las bacterias colónicas fermentan los FOS y otros carbohidratos no digeribles, aumentando la producción de AGCC (como butirato, propionato, y acetato) y otros ácidos orgánicos, como el ácido láctico. Estos AGCC bajan el pH, acidificando el contenido luminal; por consecuencia de ese $\mathrm{pH}$ menor se disuelven los compuestos insolubles de Ca tales como el fosfato, y aumenta la concentración de Ca soluble en el lumen (Ca ionizado), elevando también su absorción por difusión pasiva9, 21,24,37,38. Por otro lado, la reducción de las concentraciones de $\mathrm{Ca}$ en la dieta exacerban la acidez del lumen intestinal, pudiendo perjudicar la mucosa. El Ca actúa como buffer en la acidificación causada por la fermentación bacteriana en el intestino grueso ${ }^{39}$. De esta forma, para que tengan lugar los efectos positivos de los fructanos en la absorción de $\mathrm{Ca}$, se debe considerar la concentración de $\mathrm{Ca}$ en las raciones ${ }^{39,40}$. Es también posible que los AGCC contribuyan directamente al aumento de la absorción de Ca vía un mecanismo de intercambio catiónico y al aumento del intercambio de $\mathrm{H}+$ celular con $\mathrm{Ca}^{2}{ }^{2}$ luminal ${ }^{30}$.

-Un mecanismo vía calbindina - D9 K. Algunos estudios muestran que la ingesta de oligofructanos reduce los niveles de la proteína calbindina-D9 $\mathrm{K}$ (CaBP) en el intestino delgado, mientras que los mismos aumentan en el intestino grueso ${ }^{41,42}$. El aumento en la absorción de Ca por difusión pasiva en el colon podría accionar un mecanismo de regeneración que inhibiría la absorción activa (o transcelular) duodenal, y éste podría implicar posiblemente una disminución de Calbindina D9 K en el intestino delgado ${ }^{43}$. Otro estudio concluye que también podría aumentar la absorción transcelular, típica del intestino delgado, en el intestino grueso ${ }^{42}$.

-Con el aumento de los AGCC aumenta también la proliferación de células epiteliales, produciendo un efecto trófico en la mucosa. Algunos trabajos en animales permitieron comprobar el aumento de la profundidad de la cripta, del número de células epiteliales y hasta de las bifurcaciones en las criptas. Todo esto trae como consecuencia un aumento de la superficie de absorción $25,44,45$.

-Un estudio publicado recientemente fue el primero en mostrar cómo el consumo de FOS aumentó la expresión genética de TRPV 6 y de la proteína calbindinaD9k (proteína que transporta el Ca a través del citoplasma hasta la membrana basolateral). El estudio explica que los FOS no afectan directamente la expresión génica, y los AGCC formados en la fermentación de los FOS son considerados como probables intermediarios ${ }^{46}$.

El grado de estos efectos parece ser específico para el tipo de fructano y puede depender de la dosis ingerida ${ }^{45,47}$.

Hasta ahora no ha sido testeado el consumo de fructanos de cadena larga en ratas hembras jóvenes que se encuentran en la edad de crecimiento. Recientemente, un estudio no encontró efectos en la DMO ni en los marcadores de recambio óseo (Osteocalcina y Telepeptideo carboxi-terminal del colágeno). Sin embargo, este grupo obtuvo una reducción del $26 \%$ en el contenido de masa magra, posiblemente por una disminución en la ingesta y en el peso corporal total ${ }^{48}$. Se cree que el mecanismo por el cual se logran estos efectos es a través de la modificación de los péptidos asociados al intestino (envueltos en la regulación del apetito y de peso corporal) por los productos de la fermentación de los fructanos ${ }^{49}$.

\section{Estudios realizados en animales}

Varios estudios en los últimos años comprobaron la eficacia de los prebióticos en el aumento en la absorción de Ca. En el estudio realizado por Coudray et al. ${ }^{50}$, los autores se propusieron evaluar los grados de estimulación de distintos prebióticos. Utilizaron dietas con oligofructosa (FOS), inulina, mezcla de ambas 
(Synergy1) y otra dieta con inulina ramificada. Todos los tipos de fructanos utilizados aumentaron la fermentación, como la absorción y la retención intestinal de Mg; sin embargo, sólo la mezcla de oligofructosa (GP 4) e inulina (GP 25) mostró una diferencia significativa en el aumento de la absorción de Ca, aumentando un 25\% su retención en el hueso.

El mismo grupo de investigadores, un año después, quiso ver si el aumento de la absorción de minerales está relacionado con el período de tiempo en el que se consumió el prebiótico y cómo influían los diferentes niveles de ingesta de $\mathrm{Ca}$. A pesar de lo esperado, el efecto de la inulina en la absorción de Ca dependió de los niveles de la ingesta del mismo y de la duración del consumo de inulina. En un período corto de consumo de inulina, su efecto influyó tanto en las dietas con alta o con baja ingesta de $\mathrm{Ca}$, aunque en períodos más prolongados aumentó significativamente más la absorción en los grupos que recibían una baja concentración dietética del mineral. En suma, la eficacia de la absorción de Ca se vio afectada negativamente por sus niveles en la ingesta. Estos resultados muestran que el efecto de la inulina en la absorción de Ca puede ser más importante en los casos donde haya una baja ingesta o donde los requerimientos estén aumentados ${ }^{51}$.

Al mismo resultado llegó un estudio anterior realizado por Lemort et al.52, que observó que la inulina aumenta la absorción de Ca y la densidad mineral en el hueso cuando es consumida por largos períodos, aunque en dietas con bajas ingestas de Ca.

Para evaluar el efecto potencialmente sinérgico de una combinación de carbohidratos fermentables, se administraron diferentes dietas a 4 grupos de ratas $(n=8 \mathrm{c} / \mathrm{u})$ : un grupo recibió dieta sin fibra, otro contenía $100 \mathrm{gr} / \mathrm{kg}$ de inulina, otro $150 \mathrm{gr} / \mathrm{Kg}$ de almidón resistente (almidón de papa cruda) y el cuarto grupo obtuvo una mezcla de $50 \mathrm{gr} / \mathrm{kg}$ de inulina y $75 \mathrm{gr} / \mathrm{kg}$ de almidón resistente. Los resultados confirmaron que la inulina y el almidón resistente produjeron una considerable fermentación cecal en los 3 grupos, comparados con el grupo control. Por otra parte, ambos carbohidratos aumentaron perceptiblemente la absorción y el balance de Ca y Mg sin alterar su nivel plasmático. La combinación de los carbohidratos estudiados aumentó $(p<0,05)$ significativamente el $\mathrm{Ca}$ y el $\mathrm{Mg}$ soluble en el ciego, como también la absorción intestinal aparente y el balance de $\mathrm{Ca}^{53}$.

Continuando con la evaluación de la ingesta de fructanos de diferentes GP, un estudio demostró que el grupo de ratas que consumían inulina (GP>23) alcanzó una Densidad Mineral Ósea (DMO) y un Contenido Mineral Óseo (CMO) más altos que el grupo que consumió solo oligofructosa (GP entre 2 y 8). La excreción de fragmentos tipo 1 de colágeno (marcador de reabsorción ósea) disminuyó en todos los grupos, aunque la disminución fue más significativa en el grupo alimentado con inulina. En este estudio se demuestra que la inulina (GP>23) obtuvo el efecto más significativo en la biodisponibilidad de $\mathrm{Ca}$ evidenciado por el aumento de la absorción paracelular ${ }^{24}$.

Nzeusseu et al. ${ }^{35}$ llegaron a los mismos resultados que el estudio anterior, pero también midieron la proteína calbindina $9 K$ que participa en la absorción transcelular de $\mathrm{Ca}$, encontrando sus niveles aumentados en el intestino grueso en mayor proporción en el grupo alimentado con inulina. La diferencia en la capacidad antireabsortiva entre los dos grupos puede ser explicada por su diferente impacto en la absorción de Ca y su biodisponibilidad debido al aumento de los niveles de calbindina a nivel cecal. Esta observación explica porqué la inulina tiene un efecto más fuerte en el aumento de la DMO que la oligofructosa.

Sabiendo que la inulina es el fructano que más efectos tiene en el aumento de la biodisponibilidad del $\mathrm{Ca}$, Roberfroid et al. ${ }^{47}$ estudiaron el efecto de 3 dosis de inulina $(0 \mathrm{gr}, 5 \mathrm{gr}$ y $10 \mathrm{gr} / 100 \mathrm{gr})$ con 3 niveles de ingesta de $\mathrm{Ca}(0.2$, 0.5 y $1 \mathrm{gr}$ de $\mathrm{Ca} / 100 \mathrm{gr}$ ) en la CMO y DMO. Se obtuvo aumento tanto en el $\mathrm{CMO}(p<0,05)$ como en la DMO $(p<0,001)$ en las tres concentraciones dietéticas de $\mathrm{Ca}$. 
En otro estudio realizado por Lobo et al. (2006a), se evaluó no sólo la influencia de los FOS en la absorción de Ca sino también de Mg, tratando de verificarse también qué modificaciones se obtenían en la mineralización ósea. Durante 23 días se dieron 5\% de FOS ad libitum. Se evalúo la absorción aparente de Ca y Mg en 3 períodos durante el estudio (4to,10mo y 16 to día). Se realizaron varias pruebas físicas en el hueso y se llegó a las siguientes conclusiones ${ }^{37}$ : el consumo de FOS aumentó significativamente la absorción aparente de los minerales estudiados (Ca y Mg), aumentando también la retención de Ca en el hueso; este incremento aumentó significativamente la resistencia a fracturas en el fémur. Se observó una fuerte tendencia hacia valores más altos de todos los parámetros estudiados (carga máxima, carga de producción, tiesura, resistencia, y energía absorbida). Sin embargo, solamente los valores de carga máxima y de carga de producción obtuvieron diferencias estadísticamente significativas $(p<0,05)$.

En otro estudio realizado por los mismos autores, dividieron los animales en 3 grupos de dietas (control sin FOS, FOS 5\% y FOS 7,5\%). Se utilizó como fuente de FOS una raíz llamada yacón, que contiene un $55,3 \%$ de FOS. Se llegaron a los mismos resultados que en el estudio anterior, pero en este estudio se evaluaron las diferencias morfológicas del epitelio intestinal. Se observó un aumento del número y la profundidad de las criptas y un aumento de las bifurcaciones de las mismas, lo cual sugirió un aumento en la superficie del área de absorción del ciego, que contribuyó a la absorción mineral. Contrariamente a los efectos obtenidos en la absorción de $\mathrm{Ca}$, el consumo de yacón no afectó la absorción de magnesio (Mg), a pesar del aumento significativo observado en el balance de $\mathrm{Mg}$ en el grupo con $7,5 \%$ de FOS. La excreción urinaria de $\mathrm{Mg}$ en el grupo control fue significativamente mayor que en el grupo de ratas que consumieron $7,5 \%$ de FOS, lo que demuestra la alta retención de Mg en este último ${ }^{36}$.

Más recientemente, se estudió la variación del efecto producido por la inulina de diferentes fuentes (inulina purificada, inulina enriquecida con fructanos de cadena corta e inulina deshidratada de la achicoria). El estudio continúa mostrando el efecto benéfico de la adición de inulina a la dieta sin obtener diferencias significativas entre los tres grupos respecto a la absorción de minerales. Llamativamente, los resultados mostraron un aumento significativo del $\mathrm{CMO}$ en los animales que consumieron inulina deshidratada de la achicoria, interpretando un efecto benéfico en la diferencia del contenido de otros minerales (Mg y K) que favorecen el crecimiento óseo ${ }^{54}$.

Otros estudios sugieren evaluar la interacción de los prebióticos con otros componentes de la dieta. Por ejemplo, se ha estudiado su efecto benéfico con la adición de aceite de pescado, logrando un mayor beneficio en la absorción aparente de $\mathrm{Ca}$, Zn, Fe, Cu y un mayor contenido mineral óseo de Ca y $Z n$ en los animales alimentados con la combinación de fructanos y aceite de pescado, en comparación con los alimentados únicamente con fructanos ${ }^{44}$.

Muchos alimentos que contienen prebióticos también cuentan en su composición con ácido fítico (AF), principal responsable por la interferencia en la absorción de minerales. Varios estudios han evaluado la interacción entre ambos, AF y FOS, y se comprobó que el efecto negativo en la biodisponibilidad de minerales del AF se puede compensar con la ingesta de FOS 55,56 .

\section{Estudios realizados en humanos}

Claramente los adolescentes pertenecen a un grupo poblacional en donde el consumo de Ca es primordial para garantizar la máxima mineralización del hueso.

Las mujeres adolescentes cerca de la menarca aumentaron considerablemente la absorción de Ca con el consumo de una mezcla de inulina y oligosacáridos en un jugo de naranja con alto contenido de $\mathrm{Ca}(1500 \mathrm{mg} / \mathrm{d})^{57}$. Posteriormente, el mismo grupo de investigadores realizó un análisis multivariado en un estudio similar, 
asociando diferentes factores con el grado de significancia sobre los beneficios del consumo de inulina en la absorción de $\mathrm{Ca}$, el cual evidenció una mayor significancia con la variable baja ingesta de $\mathrm{Ca}(p=0,08)$ y baja absorción de $\mathrm{Ca}$ al inicio del estudio $(p=0,016)^{58}$.

La mayor parte de los estudios hechos en humanos hasta entonces han sido de relativo corto plazo, y no se estimó directamente el potencial benéfico de la suplementación con fructanos sobre la mineralización de los huesos. Por ello, en el 2005 fue realizado otro estudio en adolescentes, aunque esta vez el consumo de fructanos tuvo lugar durante un largo período. Se administró con el desayuno 8gr/día de fructanos (de bajo y alto GP) a 100 jóvenes con una edad media de 11 años. La absorción de Ca fue medida usando isótopos estables, en la $8 a$ semana y después de 1 año. La medición de la densidad mineral ósea y el contenido mineral en el hueso (CMO), sólo se realizó después de 1 año. Tanto a las 8 semanas $(p<0,001)$ como un año después $p=0,04$ ), hubo una diferencia significativa en la absorción de Ca en los adolescentes que recibieron la suplementación con fructanos. Después de 1 año, el grupo que recibió suplementación con fructanos obtuvo un mayor incremento en el CMO $(p=0,03)$ y en la DMO $(p=0,01)$ en comparación con el grupo control. Este estudio se realizó bajo una adecuada ingesta de $\mathrm{Ca}^{34}$. Si bien esta suplementación no mostró resultados positivos en un tercio de los participantes, más tarde los mismos autores resaltan la importancia en el efecto logrado, el cual sería comparable al aumento de la ingesta diaria de por lo menos $250 \mathrm{mgCa} /$ día en la dieta de los adolescentes ${ }^{59}$. A su vez, muestran por medio de isótopos estables cómo el $70 \%$ del aumento de la absorción de Ca sucede en el colon. Este beneficio puede ser especialmente importante cuando la absorción en el intestino delgado se ve afectada por razones anatómicas o fisiológicas ${ }^{60}$.

Continuando con la misma técnica de doble isótopo estable en adolescentes, un estudio se propuso distinguir entre el Ca exógeno y endógeno, y de esta forma, medir la verdadera absorción intestinal. Se obtuvo un aumento del $26 \%$ en la absorción de Ca en adolescentes que consumieron jugo de naranja suplementado con $5 \mathrm{gr}$ de oligofructosa (3 veces/día) ${ }^{61}$. Años más tarde, los mismos investigadores evaluaron el aumento de la absorción de minerales, realizando sin embargo un suplemento durante 36 días con FOS de cadena corta. Encontraron que no se modificó la absorción de Ca, aunque la misma aumentó un $18 \%$ la de $\mathrm{Mg}^{62}$.

En un estudio realizado en Francia en hombres jóvenes que consumieron $40 \mathrm{gr} / \mathrm{dí}$ de inulina por 26 días, se evidenció que el aumento en la absorción aparente de Ca (40\%) no influyó negativamente en la absorción de otros minerales como $\mathrm{Mg}$, Fe o $\mathrm{Zn}^{63}$. El mismo grupo poblacional obtuvo un aumento de la absorción aparente de Ca $(43 \%)$ y, por encima de todo, una mayor retención del mineral tras la ingesta de anhídrido de difructosa III (DFAllI), un disacárido no digerible extraído de la remolacha. Este estudio concluye que el aumento de la absorción de Ca a través de DFAlll, preferentemente promueve la formación ósea, más que la disminución de la reabsorción (debido al aumento de la osteocalcina más que la disminución de la Deoxipiridinolina) ${ }^{64}$.

La mayoría de los estudios en que son evaluados los principales síntomas que presenta la ingestión de FOS, señalan a la distensión abdominal y la flatulencia como los más frecuentes, sin ser estadísticamente importante la proporción de individuos que los manifiestan ${ }^{65}$.

Con el incremento de la esperanza de vida surge un aumento del número de adultos y ancianos. Esto trae aparejado un mayor gasto en salud pública para la atención de esta población. La osteoporosis, como una de las enfermedades crónicas degenerativas, se encuentra en la mira de los programas de prevención en todo el mundo, para intentar demorar el aparecimiento de complicaciones.

Todo esto ha ocasionado una mayor preocupación por la alimentación por parte de la 
población y de los organismos públicos de salud, con el intuito de investigar la utilización de alimentos en la prevención de enfermedades.

Pensando que la pérdida de Ca óseo se acentúa en la edad adulta y sobre todo en mujeres postmenopáusicas, se evaluó el efecto de la ingesta de una dosis diaria de FOS de cadena corta (10gr) por 5 semanas, cuando no estaban recibiendo terapia de reemplazo hormonal. Los resultados que se obtuvieron demostraron que no tenía ningún efecto en la absorción intestinal de $\mathrm{Ca}$, contrariando los trabajos anteriormente citados. Sin embargo, se observó que el consumo de FOS aumentaba la absorción de Ca en mujeres que habían iniciado la menopausia hace más de 6 años ${ }^{66}$. En este trabajo sólo se utilizó FOS con bajo grado de polimerización.

Al mismo grupo etario se ofreció una mezcla de fructanos de cadena corta y larga (10gr/día) durante 6 semanas. Se observó un aumento significativo en la absorción tanto de Ca como de $\mathrm{Mg}$, en comparación al grupo placebo ${ }^{67}$.

Los probióticos podrían tener efectos potenciales en el aumento del hueso independientemente a los efectos de los prebióticos. Esto ocurre por la producción de metabolitos o síntesis de vitaminas por parte la microflora intestinal, ya que se requieren varias para la formación de la matriz ósea y el crecimiento del hueso ${ }^{68}$. Se realizaron pocos estudios con la utilización exclusiva de probióticos. Un estudio evaluó la estrategia del uso de leche fermentada con probióticos para prevenir o retardar la pérdida ósea de Ca en mujeres perimenopáusicas Fue realizado al azar doble ciego con 40 pacientes mujeres (entre 40-50 años), a las que se les ofreció leche fermentada con L. paracasei SanCor CRL431 y Lactobacillus acidophilus, o leche pasteurizada no fermentada. Los resultados obtenidos indicaron diferencias significativas en los valores urinarios de Deoxipiridinolina (marcador de reabsorción ósea) entre el día 0 y el día $35(p<0,05)$ entre las mujeres que recibían probióticos y las que recibían placebo. A la vez, el N-telopéptido urinario (otro marcador de resorción ósea) y la relación $\mathrm{Ca}$ /creatinina no mostraron diferencias. Los autores concluyen que el consumo de leche fermentada disminuyó la pérdida de Ca en mujeres adultas ${ }^{69}$.

Otro estudio comprobó el aumento del efecto benéfico en los huesos de las mujeres que se encuentran en la menopausia con el agregado de probióticos (Lactobacillus casei) a una mezcla de prebióticos (isoflavona de la soja y FOS) ${ }^{70}$.

\section{CONCLUSIONES}

Luego de realizar una compilación de los artículos publicados sobre el tema, se observa que en la mayoría de los estudios se usó sólo un tipo de prebióticos, los fructanos ${ }^{71}$. Sin embargo, cabe destacar que la mayor eficiencia en la absorción de minerales se logra cuando se ofrece una mezcla de frutanos de diferentes grados de polimerización.

En la búsqueda realizada no se encontró ningún estudio clínico o de meta-análisis con el tópico de prebiótico el cual, si existiera, podría aportar importante información para ser utilizada en el inicio de investigaciones en el área clínica.

Como fue presentado, los fructanos mejoran la absorción de Ca en los adolescentes, pudiendo otorgar un mayor depósito en el hueso y optimizando el pico máximo de masa ósea ${ }^{30}$.

Se puede observar que cuando la absorción intestinal de Ca y Mg es baja, el efecto de los fructanos es mayor. Ya que el poder de absorción de Ca disminuye con la edad, el aumento relativo en la absorción inducido por los fructanos aumenta con la vejez ${ }^{72}$. Por eso hacen falta más estudios sobre los efectos entre el metabolismo del Ca y el aumento del contenido mineral en el hueso, requiriéndose más análisis de formación y resorción ósea. No obstante, los resultados de los estudios antedichos sugieren fuertemente que la adición de fructanos a los alimentos representa una oportunidad para aumentar la biodisponibilidad del Ca presente en la dieta.

Se comprueba de esta forma que la asociación entre fructanos es efectiva para 
aumentar la biodisponibilidad de Ca en un alimento, pudiendo aumentar la eficacia de absorción de uno de los minerales que presenta mayor déficit en todo el mundo.

\section{APOYOY A GRADCIMIENTO}

Conselho Nacional de Desenvolvimento Científico e Tecnológico (Becas, Procesos no. 130161/ 2008-0 y 505758/2008), Fundação de Amparo à Pesquisa do Estado de São Paulo (Auxilio a la investigación, Procesos n 2006/01735-0 y 2007/59260-0).

\section{REFERENCIAS}

1. Food and Agriculture Organization of the United Nations. Health and nutritional properties of probiotics in food including powder milk with live lactic acid bacteria. Córdoba; 2001 [cited 2005 Feb.3]. Available from: <ftp://ftp.fao.org/docrep/ fao/meeting/009/y6398e.pdf>.

2. Sanders ME. Probiotics: considerations for human health. Nutr Rev. 2003; 61:91-9.

3. Gibson GR, Roberfroid M. Dietary modulation of the human colonic microbiota: introducing the concept of prebiotics. J Nutr. 1995; 125:1401-12.

4. Reid G, Sanders ME, Rex GH, Gibson GR, Mercenier $A$, Rastall $R$, et al. New scientific paradigms for probiotics and prebiotics. J Clin Gastroenterol. 2003; 37:105-18.

5. Saad SMI. Probióticos e prebióticos: o estado da arte. Braz J Pharm Sciences. 2006; 42:1-16.

6. Buriti FCA, Cardarelli HR, Saad SMI. Biopreservation by Lactobacillus paracasei in coculture with Streptococcus thermophilus in potentially probiotic and synbiotic fresh cream cheeses. J Food Protection. 2007; 70:228-35.

7. Anjo DLC. Alimentos funcionais em angiologia e cirurgia vascular. J Vasc Br. 2004; 3:145- 54.

8. Walzem RL. Functional foods. Trends Food Sci Technol. 2004; 15:518

9. Manning TS, Gibson GR. Prebiotic. Best Practice Res Clin Gastroenterol. 2004; 18:287-98.

10. Langlands SJ, Hopkins MJ, Coleman N, Cummings $\mathrm{JH}$. Prebiotic carbohydrates modify the mucosa associated microflora of the human large bowel. Gut. 2004; 53:1610-16.

11. Roberfroid M. Prebiotics: the concept revisited. J Nutr. 2007; 137: 830S-7S.
12. Schaafsma G. Lactose and Lactose derivaties as bioactive ingredients in human nutrition. Int Diary J. 2008; 18:458-65.

13. Gibson GR, Probert HM, Van Loo JAE, Rastall RA, Roberfroid M. Dietary modulation of the human colonic microbiota: updating the concept of prebiotic. Nutr Res Rev. 2004; 17:259-75.

14. Pedreschi R, Campos D, Noratto G, Chirinos R, Cisneros-Zevallos L. Andean yacon root (Smallanthus sonchifolius Poepp. Endl) fructooligosaccharides as a potential novel source of prebiotics. J Agric Food Chem. 2003; 51:5278-84

15. Roberfroid MB, Van Loo JAE, Gibson GR. The bifidogenic nature of chicory inulin and its hydrolysis products. J Nutr. 1998; 128:11-9.

16. Fuentes-Zaragoza E, Riquelme-Navarrete MJ, Sánchez-Zapata E, Pérez-Álvarez JA. Resistant starch as funcional ingredient. Food R Int. 2010; 43:931-42

17. Bruzzese E, Volpicelli M, Squaglia M, Tartaglione A, Guarino A. Impact of prebiotics on human health. Dig. Liver Dis. 2006; 38(Suppl 2):S283-7.

18. Perrin S, Warchol M, Grill JP, Schneider F. Fermentations of fructo-oligosaccharides and their components by Bifidobacterium infantis ATCC 15697 on batch culture in semi-synthetic medium. J Appl Microbiol. 2001; 90:859-65.

19. Kaplan H, Hutkins R. Metabolism of fructooligosaccharides by Lactobacillus paracasei 1195. Appl Environ Microbiol. 2003; 69:2217-22.

20. Rossi M, Corradini C, Amaretti A, Nicolini M, Pompei A, Zanoni S, et al. Fermentation of Fructooligosaccharides and Inulin by Bifidobacteria: a comparative study of pure and fecal cultures. Appl Environ Microbiol. 2005; 71:6150-8.

21. Rastall R, Gibson GR, Gill HS, Guarner F, Klaenhammer TR, Pot B, et al. Modulation of the microbial ecology of the human colon by probiotics, prebiotics and synbiotics to enhance human health: an overview of enabling science and potential applications. FEMS Microbiol Ecol. 2005; 52: 145-52.

22. Roberfroid M. Introducing inulin-type fructans. $\mathrm{Br}$ J Nutr. 2005; 93(Suppl 1):S13-S25

23. Organización Mundial de la Salud. Comité de expertos y grupos de estudio. Prevención y tratamiento de la osteoporosis. Ginebra; 2000. Available from: <http://www.who.int/gb/ebwha/ pdf_files/EB114/B114_13-sp.pdf>.

24. Kruger MC, Brown KE, Collett G, Layton L, Schollum LM. The effect of fructooligosaccharides with various degrees of polymerization on calcium bioavailability in the growing rat. Exp Biol Med. 2003; 228:683-8. 
25. Raschka L, Daniel H. Mechanisms underlying the effects of inulin-type fructans on calcium absorption in the large intestine of rats. Bone. 2005;37:728-35.

26. Pérez-Conesa D, Lopez GY, Ros G. Effects of probiotic, prebiotic and synbiotic follow-up infant formulas on large intestine morphology and bone mineralisation in rats. J Sci Food Agric. 2007; 87: 1059-68.

27. Weaver C. Inulin, oligofructose and bone health: experimental approaches and mechanisms. Br J Nutr. 2005;93(Suppl 1):S99-S103.

28. Peng JB, Brown, EM, Hediger MA. Apical entry channels in calcium-transporting epithelia. News Physiol Sci. 2003; 18:158-63.

29. Machado DF, Silva RR, Fanchiotti FE, Costa NMB. Probióticos, prebióticos e simbióticos e seus efeitos na biodisponibilidade do cálcio. J Braz Soc Food Nutr. 2001; 22:73-83.

30. Coxam V. Inulin-type fructans and bone health: state of the art and perspectives in the management of osteoporosis. Br J Nutr. 2005; 93(Suppl 1):S111-S23.

31. Mineo $H$, Hara $H$, Kikuchi $H$, Sakurai $H$, Tomita F. Various indigestible saccharides enhance net calcium transport from the epithelium of the small and large intestine of rats in vitro. J Nutr. 2001; 31:3243-46.

32. Zafar TA, Weaver CM, Zhao Y, Martin BR, Wastney E. Nondigestible Oligosaccharides increase calcium absorption and suppress bone resorption in ovariectomized rats. J Nutr. 2004; 134:399-402.

33. Roberfroid M, Cump J, Devogelaer P. Dietary chicory inulin increases whole-body bone mineral density in growing male rats. J Nutr. 2002; 132:3599-602.

34. Abrams AA, Griffin IJ, Hawthorne KM, Liang L, Gunn SK, Darlington $G$, et al. A combination of prebiotic short- and long-chain inulin-type fructans enhances calcium absorption and bone mineralization in young adolescents. Am J Clin Nutr. 2005; 82:471-6.

35. Nzeusseu A, Dienst D, Haufroid V, Depresseux G, Devogelaer JP, Manicourt DH. Inulin and fructooligosaccharides differ in their ability to enhance the density of cancellous and cortical bone in the axial and peripheral skeleton in growing rats. Bone. 2006; 37:394-9.

36. Lobo AR, Colli C, Alvares EP, Filisetti TMCC. Effects of fructans-containing yacon (Smallanthus sonchifolius Poepp \& Endl.) flour on caecum mucosal morphometry, calcium and magnesium balance, and bone calcium retention in growing rats. Br J Nutr. 2007; 97:776-85.
37. Lobo AR, Colli C, Filisetti TMCC. Fructooligosaccharides improve bone mass and biomechanical properties in rats. Nutr Res. 2006; 26:413-20.

38. Pérez-Conesa D, López G, Abellán P, Ros G. Bioavailability of calcium, magnesium and phosphorus in rats fed probiotic, prebiotic and synbiotic powder follow-up infant formulas and their effect on physiological and nutritional parameters. J Sci Food Agric. 2006; 86:2327-36.

39. Lobo AR, Cocato ML, Jorgetti V, Sá LR, Nakano EY, Colli C. Changes in boné mass, biomechanical properties, and microarchitecture of calcium-and iron-deficient rats fed diets supplemented with inulin-type fructans. Nutr Res. 2009; 29(12):87381.

40. Rémésy C, Levrat MA, Gamet L, Demigné C. Cecal fermentations in rats fed oligosaccharides (inulin) are modulated by dietary calcium level. Am J Physiol. 1993; 264: G855-G62.

41. Sakuma K. Molecular mechanism of the effect of fructooligosaccharides on calcium absorption. Biosci Microflora. 2002; 21:13-20.

42. Ohta A, Motohashi Y, Ohtsuki M, Hirayama M, Adachi T, Sakuma K. Dietary fructooligosaccharides change the concentration of calbindin-D9k differently in the mucosa of the small and large intestine of rats. J Nutr. 1998; 128:934-9.

43. Takasaki M, Inaba H, Ohta A, Motohashi Y, Sakai $\mathrm{K}$, Morris $\mathrm{H}$, et al. Dietary short-chain fructooligosaccharides increase calbindin- D9K levels only in the large intestine in rats independent of dietary calcium deficiency or serum 1,25 dihydroxyvitamin D levels. Int J Vitaminol Nutr Res. 2000; 70:206-13.

44. Lobo AR, Mancini-Filho J, Alvares EP, Cocato ML, Colli C. Effects of dietary lipid composition and inulin-type fructans on mineral bioavailability in growing rats. Nutrition. 2009; 25:216-25.

45. Scholz-Ahrens KE, Schrezenmeir J. Inulin, oligofructose and mineral metabolism: experimental data and mechanism. Br J Nutr. 2002; 87(Suppl 2):S179-86.

46. Fukushima A, Aizaki Y, Sakuma K. Short-Chain fatty acids induce intestinal transient receptor potential vanilloid type 6 expression in rats and caco-2 cells. J Nutr. 2009; 139:20-5.

47. Roberfroid M. Functional food concept and its application to prebiotics. Digestive Liver Dis. 2002; 34 (Suppl 2):105-10.

48. Jamieson AJ, Ryz NR, Taylor CG, Weiler HA. Dietary long-chain inulin reduces abdominal fat but has no effect on bone density in growing female rats. Br J Nutr. 2008; 100:451-9. 
49. Cani PD, Neyrinck AM, Maton N, Delzenne NM. Oligofructose promotes satiety in rats fed a highfat diet: involvement of glucagon-like peptide-1. Obes Res. 2005; 13:1000-7.

50. Coudray C, Tressol JC, Gueux E, Rayssiguier Y. Effect of inulin-type fructans of different chain length and type of branching on intestinal absorption and balance of calcium and magnesium in rats. Eur J Nutr. 2003; 42:91-9.

51. Coudray C, Feillet-Coudray C, Tressol JC, Gueux E, Thien $S$, Jaffrelo $L$, et al. Stimulatory effect of inulin on intestinal absorption of calcium and magnesium in rats is modulated by dietary calcium intakes short- and long-term balance studies. Eur J Nutr. 2005; 44:293-302.

52. Lemort C, Roberfroid M, Devogelaer JP. Influence of chicory inulin on whole body bone mineral density in growing male rats. J Nutr. 2002; 132: 3599-602.

53. Younes $H$, Coudray C, Bellanger J, Demigné C, Rayssiguier $Y$, Rémésy $C$. Effects of two fermentable carbohydrates (inulin and resistant starch) and their combination on calcium and magnesium balance in rats. Br J Nutr. 2001;86: 479-85.

54. Demigné $C$, Jacobs $H$, Moundras C, Davicco MJ, Horcajada MN, Bernalier A, et al. Comparison of native or reformulated chicory fructans, or nonpurified chicory on rat cecal fermentation and mineral metabolism. Eur J Nutr. 2008; 47:366-74.

55. Wang Y, Zeng T, Wang S, Wang Q, Yu HX. Fructooligosaccharides enhance the mineral absorption and counteract the adverse effects of phytic acid in mice. Nutrition. 2009; doi: 10.1016/j.nut.2009.0 4.014.

56. Lopez HW, Coudray C, Levrat-Verny M-A, Coudray CF, Demigné C, Rémésy $C$. Fructooligosaccharides enhance mineral apparent absorption and counteract the deleterious effects of phytic acid on mineral homeostasis in rats. J Nutr Biochem. 2000; 11:500-8.

57. Griffin IJ, Abrams S, Hicks P, Heaney R. NonDigestible Oligosaccharides (NDO) increase calcium absorption, especially those whose calcium absorption is poorest. Pediatr Res. 2002; 51:188A.

58. Griffin IJ, Penni MD, Hicks P, Heaney R, Abrams S. Enriched chicory inulin increases calcium absorption mainly in girls with lower calcium absorption. Nutr Res. 2003;23:901-9.

59. Abrams SA, Grifn IJ, Hawthorne KM. Young adolescents who respond to an inulin-type fructan substantially increase total absorbed calcium and daily calcium accretion to the skeleton. J Nutr. 2007; 137:2524S-6S

60. Abrams SA, Hawthorne KM, Aliu O, Hicks PD, Chen Z, Grifn IJ. An inulin-type fructan enhances calcium absorption primarily via an effect on colonic absorption in humans. J Nutr. 2007; 137:2208-12.
61. Van den Heuvel EGHM, Muys T, Van dokkum M, Schaafsma G. Oligofructose stimulates calcium absorption in adolescents. Am J Clin Nutr. 1999; 69:544-8.

62. Van den Heuvel EGHM, Muijs T, Brouns F, Hendriks HFJ. Short-chain fructo-oligosaccharides improve magnesium absorption in adolescent girls with a low calcium intake. Nutr Res. 2009; 29:229-37.

63. Coudray C, Bellanger J, Castiglia-Delavaud C, Vermorel M, Rayssignuier Y. Effect of soluble or partly soluble dietary fibres supplementation on absorption and balance of calcium, magnesium, iron and zinc in healthy young man. Eur J Clin Nutr. 1997; 51:375-80.

64. Tomita K, Shiomi T, Okuhara Y, Tamura A, Shigematsu N, Hara $\mathrm{H}$. Ingestion of difructose anhydride III enhances absorption and retention of calcium in healthy men. Biosc Biotecnol Biochem. 2007; 71:681-7.

65. Bruggencate SIMT, Bovee-Oudenhoven IMJ, Lettink-Wissink MLG, Katan MB, van der Meer R. Dietary fructooligosaccharides affect intestinal barrier function in healthy men. J Nutr. 2006; 136: 70-74.

66. Tahiri M, Tressol JC, Arnaud J, Bornet FRJ, Bouteloup-Demange C, Feillet-Coudray C, et al. Effect of short-chain fructooligosaccharides on intestinal calcium absorption and calcium status in postmenopausal women: a stable-isotope study. Am J Clin Nutr. 2003; 77:449-57.

67. Holloway L, Moynihan S, Abrams SA, Kent K, Hsu $A R$, Friedlander AL. Effects of oligofructoseenriched inulin on intestinal absorption of calcium and magnesium and bone turnover markers in postmenopausal women. Br J Nutr. 2007; 97: 365-72.

68. Scholz-Ahrens KE, Schrezenmeir J. Inulin and oligofructose and mineralmetabolism: the evidence from animal trials. J Nutr. 2007; 137:2513S-23S.

69. Chaila Z, Ortiz Zavalla J, Alarcón O, Gusils C, Gauffin Cano P, Moreno R, et al. Relation between probiotic milk administration and some bone turnover markers. J Food Technol. 2005; 3:135-42.

70. Mathey J, Mardon J, Fokialakis N, Puel J, KatiCoulibaly S, Mitakou S, et al. Modulation of soy isoflavones bioavailability and subsequent effects on bone health in ovariectomized rats: the case for equol. Osteoporos Int. 2007; 18:671-9.

71. Van Loo JA. Prebiotics promote good health: the basis, the potential, and the emerging evidence. J Clin Gastroenterol. 2004; 38(Suppl 6):S70-5.

72. Roberfroid M. Inulin-type fructans: functional food ingredients. Trends Food Sci Technol. 2006; 17:39-41.

Recibido el: 25/11/2009

Versión final re-apresentada el: 22/7/2010 Aprobado el: 23/9/2010 